\title{
Religiosität in unterschiedlichen Religionskulturen - Vergleiche auf der Basis der Centrality of Religiosity Scale
}

\author{
Stefan Huber $(\mathbb{D} \cdot$ Michael Ackert $(\mathbb{D} \cdot$ Herbert Scheiblich
}

Eingegangen: 4. Juni 2020 / Angenommen: 9. Juli 2020 / Online publiziert: 7. August 2020

(C) Der/die Autor(en) 2020

Zusammenfassung Der Beitrag diskutiert die interreligiöse Anwendbarkeit der Centrality of Religiosity Scale (CRS). Ein wesentlicher Teil besteht in der soziologischen Begründung von sechs Kerndimensionen der Religiosität (Ideologie, Intellekt, Erfahrung, private Praxis, öffentliche Praxis, Konsequenzen im Alltag), wobei die CRS auf den ersten fünf Dimensionen basiert. Bei der theoretischen Begründung der Kerndimensionen spielen ihre relative Autonomie sowie soziale Erwartungen an religiöse Personen eine entscheidende Rolle. Für die interreligiöse Anwendbarkeit der CRS spricht, dass die thematisierten sozialen Erwartungen bei allen größeren Religionskulturen gegeben sind. Darauf aufbauend werden die Operationalisierung der Kerndimensionen sowie einzelne Modifikationen der Indikatoren in bestimmten Religionskulturen dargestellt. Dabei sind die Formspezifik und inhaltliche Allgemeinheit der Indikatoren zentral, um die CRS vergleichend in unterschiedlichen Religionskulturen anwenden zu können. In empirischer Hinsicht wird die Reliabilität (Cronbachs Alpha) der aus zehn Indikatoren bestehenden CRS-10 bei jüdischen, christlichen, islamischen, hinduistischen und buddhistischen Respondenten in den Daten des internationalen Religionsmonitors 2007, der in 21 Ländern unter Berücksichtigung der genannten fünf großen Religionskulturen durchgeführt wurde,

\footnotetext{
S. Huber $(\bowtie)$

Institut für Empirische Religionsforschung, Theologische Fakultät, Universität Bern,

Länggassstrasse 51, 3012 Bern, Schweiz

E-Mail: stefan.huber@theol.unibe.ch

M. Ackert

Departement für Psychologie, Universität Fribourg, Rue Petermann-Aymon-de-Faucigny 2, 1700 Fribourg, Schweiz

E-Mail: michael.ackert@unifr.ch

H. Scheiblich

Institut für Empirische Religionsforschung, Theologische Fakultät, Universität Bern,

Länggassstrasse 51, 3012 Bern, Schweiz

E-Mail: herbert.scheiblich@theol.unibe.ch
} 
diskutiert. Dabei wurde deutlich, dass sich nach der Berücksichtigung des Modernisierungsgrads einer Gesellschaft und der Anteil von Säkularen die Reliabilitätskoeffizienten in allen Religionskulturen in einem akzeptablen Bereich befinden.

Schlüsselwörter Religiosität · Spiritualität · Kerndimension · Interreligiöse Vergleichbarkeit · Tertium comparationis

\title{
Religiosity in various religious cultures: comparisons based on the Centrality of Religiosity Scale
}

\begin{abstract}
The article discusses the interreligious applicability of the Centrality of Religiosity Scale (CRS). A substantial part consists in the sociological justification of six core dimensions of religiosity (ideology, intellect, experience, private practice, public practice, consequences in everyday life), whereby the CRS is based on the first five dimensions. In the theoretical justification of the core dimensions, their relative autonomy and social expectations of religious persons play a determining role. The interreligious applicability of the CRS is supported by the fact that the social expectations addressed are present in all major religious cultures. Based on this, the operationalization of the core dimensions and individual modifications of the indicators in certain religious cultures are presented. The form specificity and the general nature of the indicators in terms of content are central to being able to apply the CRS in different religious cultures in a comparative way. From an empirical point of view, the reliability (Cronbach's Alpha) of the CRS-10, consisting of ten indicators in Jewish, Christian, Islamic, Hindu and Buddhist respondents, is discussed in the data of the International Religion Monitor 2007, which was conducted in 21 countries, taking into account the five major religious cultures mentioned above. It became clear that after taking into consideration the degree of modernization of a society and the proportion of secular people, the reliability coefficients in all religious cultures are within an acceptable range.
\end{abstract}

Keywords Religiosity - Spirituality · Core dimension · Interreligious comparison · Tertium comparationis

Résumé L'article traite de l'applicabilité interreligieuse de la «Centrality of Religiosity Scale» (CRS). Une partie essentielle consiste en la justification sociologique de six dimensions fondamentales de la religiosité (idéologie, intellect, expérience, pratique privée, pratique publique, conséquences dans la vie quotidienne), sorte que le CRS se fonde sur les cinq premières dimensions. Dans la justification théorique des dimensions fondamentales, leur autonomie relative et les attentes sociales des personnes religieuses jouent un rôle décisif. L'applicabilité interreligieuse de la CRS est soutenue par le fait que les attentes sociales visées sont présentes dans toutes les grandes cultures religieuses. Sur cette base, l'opérationnalisation des dimensions fondamentales et les modifications individuelles des indicateurs dans certaines cultures religieuses sont présentées. La spécificité de la forme et la généralité des indicateurs en termes de contenu sont essentielles pour l'application comparative du CRS dans différentes cultures religieuses. D'un point de vue empirique, la fiabilité 
(Alpha de Cronbach) du CRS-10, composé de dix indicateurs parmi les répondants juifs, chrétiens, islamiques, hindous et bouddhistes, est examinée dans les données de l'International Religion Monitor 2007, qui a été réalisé dans 21 pays, en tenant compte des cinq grandes cultures religieuses mentionnées ci-avant. Il est apparu clairement qu'après avoir pris en compte le degré de modernisation d'une société et la proportion de laïcs, les coefficients de fiabilité dans toutes les cultures religieuses se situent dans une marge acceptable.

Religion und Kultur sind auf vielfache Weise aufeinander bezogen und miteinander verschränkt. In unterschiedlichen Kulturen nehmen Religionen unterschiedliche Gestalten an. Aus diesem Grund ist es zum Beispiel sinnvoll, zwischen einem indonesischen und arabischen Islam oder einem lateinamerikanischen und westeuropäischen Christentum zu unterscheiden. Sollen vergleichende empirische Untersuchungen zwischen Angehörigen ,gleicher“ Religionen in unterschiedlichen Kulturen oder Angehörigen unterschiedlicher Religionen in gleichen oder unterschiedlichen Kulturen durchgeführt werden, dann stellt sich die Frage nach einem tertium comparationis, das den vergleichenden Studien zugrunde liegt (Huber und Krech 2009). Dieses ,zeichnet sich notwendigerweise durch eine hinreichende Abstraktion aus, um heterogene empirische Sachverhalte miteinander auf Unterschiede und Gemeinsamkeiten hin vergleichen zu können. Wenn aber das komparatistische Verfahren nicht aus einem reinen Kunstgriff bestehen soll, mit dem an die Empirie ausschließlich ihr äußerliche Elemente herangetragen werden, wenn die tertia comparationis also mit dem Material korrespondieren sollen, dann müssen bestimmte Aspekte einseitig hervorgehoben und aus der Empirie abstrahiert werden“" (Huber und Krech 2009, S. 55).

Der vorliegende Beitrag geht der Frage nach, ob die Centrality of Religiosity Scale (CRS) ein brauchbares tertium comparationis für interreligiöse und interkulturelle Vergleiche darstellt. Dies wird zunächst theoretisch in Bezug auf die Konstruktionsprinzipien der Skala diskutiert. Dabei ist die entscheidende Frage, ob die Kerndimensionen der Religiosität, die in der Skala postuliert werden, an die großen religiösen Traditionskomplexe (z. B. Buddhismus und Islam) nur äußerlich herangetragen werden oder ob sie aus ihnen abstrahiert werden können. Darauf aufbauend wird die Anwendbarkeit der Skala auf der Basis von empirischen Ergebnissen des internationalen Religionsmonitors 2007 diskutiert. Dabei spielt das Kriterium der statistischen Reliabilität oder Zuverlässigkeit der Skala eine entscheidende Rolle.

Die CRS wurde von Huber (2003) als eine Synthese der Messmodelle von Gordon Allport und Charles Glock entwickelt. Die Skala basiert auf fünf von Stark und Glock (1970) definierten Kerndimensionen der Religiosität, die formspezifisch operationalisiert werden. Darüber hinaus wird bei der Operationalisierung der Skala darauf geachtet, dass die religiösen Inhalte möglichst allgemein formuliert sind, sodass sie in unterschiedlichen religiösen Kulturen verständlich sind.

Die erste Version der Skala (CRS-10) umfasst zehn Items (vgl. Huber 2003, 2007). Ein Jahr später wurde eine 15 Items umfassende Version (CRS-15) publiziert (vgl. Huber 2004). Bereits diese Versionen hatten den Anspruch, interreligiös gültig zu sein. Im Kontext des internationalen Religionsmonitors der Bertelsmann 


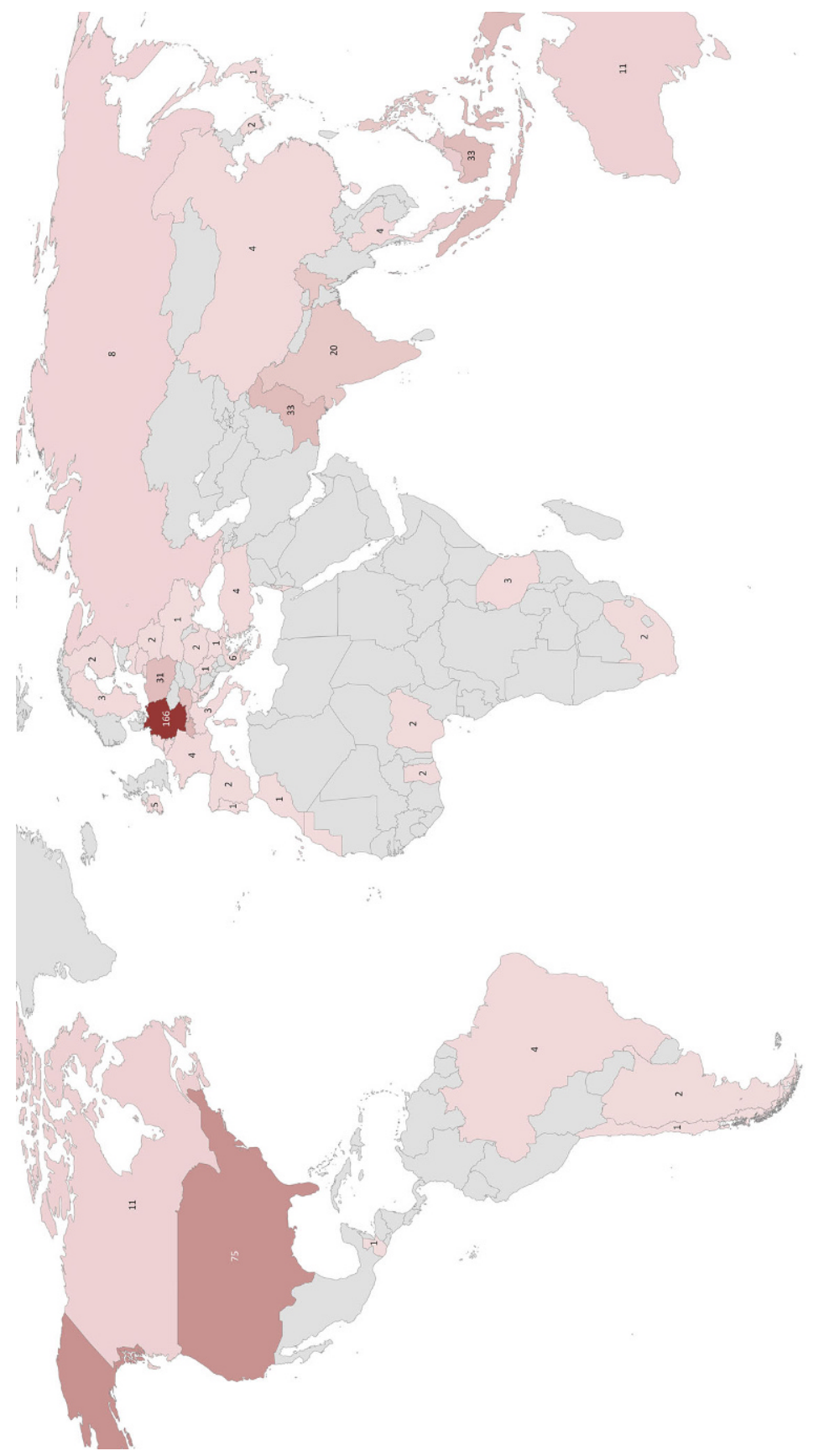

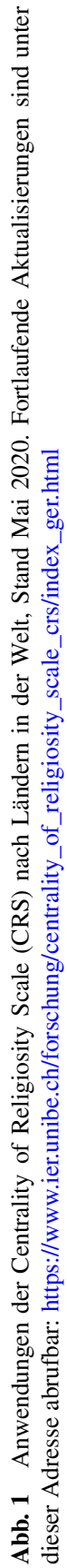


Stiftung wurde die interreligiöse Anwendbarkeit der CRS durch die Hinzufügung weiterer Versionen ausgebaut (Huber 2008a, 2009). Bei diesen Versionen, die mit dem Index „i“ (für interreligiös) gekennzeichnet sind (CRSi-7, CRSi-14, CRSi-20), wird zusätzlich zum Gebet auch nach Meditation gefragt sowie zusätzlich zu interaktiven Erfahrungen (z.B. ein Eingreifen Gottes oder des Göttlichen) werden auch partizipative Erfahrungen (z.B. das Gefühl mit allem eins zu sein) berücksichtigt. Eine systematische Darstellung aller Versionen der CRS findet sich bei Huber und Huber (2012).

In den 17 Jahren seit der Erstveröffentlichung der CRS sind 610 Anwendungen dieser Skala dokumentiert (vgl. Abb. 1). Davon entfallen auch zahlreiche Anwendungen auf Länder, die durch nicht-christliche Religionskulturen geprägt sind (Islam: 106, Judentum: 3, Buddhismus: 11, Hinduismus: 18). Bereits diese breite interreligiöse Rezeption der CRS kann als ein Indiz für die interreligiöse Anwendbarkeit der Skala gewertet werden. Zumindest gingen die Autoren und Autorinnen der Studien der These, dass die CRS in ihrem religionskulturellen Kontext zu gültigen Ergebnissen führen sollte, nach. Eine - leider noch ausstehende - systematische Metaanalyse dieser Anwendungen könnte diese Erwartungen empirisch überprüfen. Eine derartige Metaanalyse können wir im Rahmen dieses Beitrags nicht leisten. Daher gehen wird der Frage der interreligiösen Anwendbarkeit der CRS vor allem theoretisch nach. Dazu werden in Abschn. 2 die Theorie und die Operationalisierung der CRS im Detail dargestellt. Dabei spielt das Konzept von religiösen Kerndimensionen, die auf sozialen Erwartungen gründen und die für alle großen Religionskulturen postuliert werden können, eine zentrale Rolle. Die Kerndimensionen (Ideologie, Intellekt, Erfahrung, private Praxis, öffentliche Praxis, Konsequenzen im Alltag) und ihre interreligiös sensible Operationalisierung werden der Reihe nach besprochen. In Abschn. 3 wird dann die Reliabilität (Cronbachs Alpha) der aus zehn Indikatoren bestehenden CRS-10 bei jüdischen, christlichen, islamischen, hinduistischen und buddhistischen Respondenten in den Daten des internationalen Religionsmonitors 2007, der in 21 Ländern unter Berücksichtigung der genannten fünf großen Religionskulturen durchgeführt wurde, diskutiert. Einige Empfehlungen für zukünftige Forschungen runden den Beitrag ab.

\section{Theorie und Operationalisierung}

\subsection{Begriff der Religiosität}

Die CRS basiert auf einem substanziellen Begriff der Religiosität (vgl. Pollack 2000). Dieser Begriff wird jedoch nicht theistisch oder gar monotheistisch verengt, sondern er wird möglichst weit gefasst und soll explizit auch für neuere Formen der Spiritualität offen sein. In dieser Perspektive besteht das wesentliche Merkmal religiösen Erlebens und Verhaltens in einem sinnstiftenden Bezug zu einer als höher empfundenen Wirklichkeit, der eine wesentliche Relevanz für das eigene Leben zugesprochen wird. In der Religionsgeschichte wird diese höhere Wirklichkeit oft als Gott, Göttliches, höhere Macht, Geist oder spirituelle Welt benannt. Diesen Bestimmungen ist gemeinsam, dass sie den Horizont von Alltagspraktiken und deren 
naturwissenschaftlich fundierte Kontrolle überschreiten. In ähnlicher Weise spricht z.B. Martin Riesebrodt von einer ,religiösen Prämisse“ (Riesebrodt 2007, S. 115) als dem konstitutiven Merkmal religiöser Praxis.

\subsection{Kerndimensionen der Religiosität}

In der CRS wird die sinnstiftende Beziehung zu einer als höher empfundenen Wirklichkeit in fünf Kerndimensionen ausdifferenziert. Der Begriff der Kerndimension (,,core dimension“) stammt von Charles Glock (1962). Um den Status einer Kerndimension zu erlangen muss ein Merkmal nach Glock mindestens zwei Eigenschaften aufweisen - es sollte allgemein und relativ autonom sein:

- Allgemeinheit bedeutet in diesem Kontext, dass das Merkmal in allen größeren religiösen Kulturen vorkommen sollte. Diese Eigenschaft kann religionsgeschichtlich überprüft werden.

- Relative Autonomie bedeutet, dass dieses Merkmal nicht auf andere Merkmale zurückgeführt werden kann; d.h. es darf nicht redundant sein. Diese Eigenschaft kann durch statistische Analysen von quantitativen Fragebogendaten überprüft werden.

In dem Beitrag von 1962 definierte er folgende fünf Kerndimensionen der Religiosität: Intellekt, Ideologie, Erfahrung, Praxis, Konsequenzen im Alltag. Sechs Jahre später modifizierte er dieses Modell, indem er die Dimension der Konsequenzen im Alltag herausnahm und dafür die Praxis in private und öffentliche Praxis als zwei eigenständige Kerndimensionen ausdifferenzierte (vgl. Stark und Glock 1970). In seiner endgültigen Gestalt bestand das Modell von Glock somit aus den folgenden fünf Kerndimensionen: Ideologie, Intellekt, Erfahrung, private Praxis, öffentliche Praxis. Aus diesen Kerndimensionen setzt sich auch die CRS zusammen.

Alle Kerndimensionen gründen nach Glock (1962) in sozialen Erwartungen, denen Personen, die einer Religionsgemeinschaft angehören, ausgesetzt sind. Diese Erwartungen konstituieren soziale Formen, in deren Bahnen Religiosität eine bestimmte Gestalt gewinnt. Diese Formen haben sich in einem langen religionsgeschichtlichen Prozess herausgebildet. Zugleich werden sie durch die auch gegenwärtig wirkenden Erwartungen immer wieder von Neuem stabilisiert. Dabei sollte beachtet werden, dass die Formen nur ein strukturierendes Gerüst bilden. Die konkreten Gestalten des Religiösen, die sich innerhalb dieser Formen entwickelt haben und weiterentwickeln, sind vielfältig. Daher sollten diese konkreten Gestalten des Religiösen nicht mit den Formen verwechselt werden.

Von den abstrakten sozialen Formen der Kerndimensionen sollte ihre Operationalisierung strikt unterschieden werden. Dafür kommen im Prinzip alle konkreten religiösen Fragen, Einstellungen, Wahrnehmungen, Praktiken und Alltagsregeln, die in einer religiösen Kultur vorkommen, in Betracht. Bei der Operationalisierung der CRS wurde darauf geachtet, diese Dimensionen möglichst formspezifisch und inhaltlich allgemein zu operationalisieren (vgl. Huber 2003, S. 224-231). Formspezifik bedeutet, dass die soziale Form einer Kerndimension möglichst präzise und ohne Überschneidungen mit anderen Formen operationalisiert wird. Beispielsweise sollte nur die ideologische Kerndimension als Einstellung operationalisiert werden und 
die Kerndimension der religiösen Erfahrung sollte sich nur auf die Wahrnehmung von Ereignissen beziehen. Demgegenüber läge eine grobe Verletzung des Prinzips der Formspezifik vor, wenn versucht würde, die Dimension der religiösen Erfahrung über Einstellungsitems zu operationalisieren. Denn in diesem Fall bliebe unklar, ob Erfahrung oder Ideologie operationalisiert ist. Inhaltliche Allgemeinheit bedeutet, dass theologische Inhalte, die in den Indikatoren vorkommen, möglichst allgemein gehalten werden. Dadurch soll erreicht werden, dass theologische Inhalte hinter der Kerndimension zurücktreten und nicht die Zustimmung zu einem bestimmten Inhalt, sondern nur die persönliche Wichtigkeit einer Kerndimension erfasst wird. Diese Vorgabe würde beispielsweise verletzt, wenn in dem Indikator der ideologischen Dimension ein bestimmtes Gottesbild transportiert würde.

\subsubsection{Religiöse Ideologie}

Diese Kerndimension wird soziologisch durch die soziale Erwartung konstituiert, dass Angehörige von Religionsgemeinschaften an die Existenz einer höheren Wirklichkeit glauben und dass sie bestimmte Vorstellungen vom Wesen dieser Wirklichkeit haben. In der Definition der Religiosität wurde festgehalten, dass die als höher empfundene Wirklichkeit den Horizont von Alltagspraktiken und deren naturwissenschaftlich fundierten Kontrolle überschreitet. Gerade deshalb muss sie immer wieder neu postuliert und sozial abgestützt werden. Der Glaube an eine höhere Wirklichkeit findet sich in allen großen Religionen. Davon sind auch buddhistische Traditionen nicht ausgenommen. So finden sich in der buddhistischen Volksfrömmigkeit vielfache Vorstellungen von höheren Wesen und Mächten, die einen Einfluss auf die menschliche Lebenswelt ausüben - z. B. die Verehrung von Bodhisattvas (vgl. Schlieter 2001, S. 92). Grundlegender ist, dass der Buddhismus mit Karmakonzepten operiert (z.B. Samsara, Nirwana - vgl. Schlieter 2001; von Brück 2007), in denen Ereignisse der Lebenswelt durch eine ihr zugrunde liegende spirituelle Wirklichkeit erklärt werden. Damit ist das zentrale Definitionsmerkmal einer höheren Wirklichkeit gegeben. Der Unterschied gegenüber monotheistischen Religionen besteht lediglich im Wesen dieser Wirklichkeit. Sie ist im Buddhismus nicht um einen Gott zentriert, sondern wird als eine Art spirituelle Gesetzmäßigkeit vorgestellt.

In der CRS ist diese Kerndimension je nach Version durch bis zu drei Indikatoren operationalisiert (vgl. Huber und Huber 2012, S. 718):

1. Wie stark glauben Sie daran, dass Gott oder etwas Göttliches existiert?

2. Wie stark glauben Sie daran, dass es ein Leben nach dem Tod gibt - z. B. Unsterblichkeit der Seele, Auferstehung von den Toten oder Reinkarnation?

3. Wie hoch ist Ihrer Ansicht nach die Wahrscheinlichkeit, dass es eine höhere Macht gibt?

Bei allen drei Indikatoren werden Einstellungen abgefragt, was der Spezifik der ideologischen Form entspricht. Inhaltlich sind sie allgemein gehalten und beziehen sich auf zentrale Aspekte, die auf die Existenz einer höheren Wirklichkeit verweisen. Die interreligiöse Verständlich- und Anwendbarkeit der Indikatoren wird durch die Präsentation alternativer Konzepte (z. B. Gott und Göttliches oder Unsterblichkeit, Auferstehung und Reinkarnation) erreicht. In hinduistischen oder buddhistischen 
Kontexten wird empfohlen, im ersten Indikator als dritte Alternative Gottheiten zu ergänzen (vgl. Huber und Huber 2012, S. 719). Daneben wäre es auch möglich, beim ersten Indikator auch noch das Konzept von Geistern der Ahnen hinzuzufügen. Dies könnte sich beispielsweise im Konfuzianismus oder im japanischen Shinto anbieten.

\subsubsection{Religiöse Intellektualität}

Diese Kerndimension wird soziologisch durch die soziale Erwartung konstituiert, dass Angehörige von Religionsgemeinschaften über Kenntnisse ihrer Religion verfügen und daher in der Lage sind, darüber Auskunft zu geben. Daher wird in allen großen Religionen die Vermittlung religiösen Wissens gepflegt. Daneben wird die intellektuelle Dimension der Religiosität auch immer dann aktiviert, wenn das sinnstiftende Potenzial religiöser Konzepte von einem Individuum in einer konkreten Lebenssituation in Anspruch genommen wird. Denn dazu ist eine Vermittlung der konkreten Lebensumstände mit allgemeinen religiösen Konzepten notwendig. Dadurch werden die in dieser Situation relevanten Konzepte konkretisiert und mit persönlichen Erfahrungen angereichert. Es kann angenommen werden, dass dies im Leben religiöser Menschen sehr oft vorkommt. Daher handelt es sich um eine wichtige Dimension der Religiosität. Umso bedauerlicher ist es, dass sie in vielen empirischen Studien komplett ausgeblendet wird.

In der CRS ist diese Kerndimension je nach Version durch bis zu drei Indikatoren operationalisiert (vgl. Huber und Huber 2012, S. 718):

1. Wie oft denken Sie über religiöse Fragen nach?

2. Wie stark interessieren Sie sich dafür, mehr über religiöse Themen zu erfahren?

3. Wie oft informieren Sie sich durch Radio, Fernsehen, Internet, Zeitschriften oder Bücher über religiöse Fragen?

In den drei Indikatoren wird nach der Häufigkeit und der Stärke des Interesses an einer Auseinandersetzung mit religiösen Themen gefragt. Beide Aspekte zielen direkt ins Zentrum der intellektuellen Dimension. Für die interreligiöse Anwendbarkeit dieser Indikatoren spricht, dass in ihnen keine religiösen Themen vorgegeben werden. Daher ist keinerlei inhaltliche Konfundierung mit Themen, die von einer oder mehreren religiösen Traditionen bevorzugt werden, gegeben. Der einzige Inhalt ist der Begriff religiös, der auf das Ganze des Religiösen verweist.

\subsubsection{Religiöse Erfahrung}

Diese Kerndimension wird soziologisch durch die soziale Erwartung konstituiert, dass Angehörige von Religionsgemeinschaften Erlebnisse haben, in denen sich eine Art Kontakt mit der höheren Wirklichkeit, an die sie glauben, ereignet. Stark und Glock (1970, S. 126) sprechen diesbezüglich von ,some kind of direct contact to an ultimate reality“. Das Religiöse erscheint hier in der Form von sozial vermittelten und geteilten Wahrnehmungsmustern. Da diese Wahrnehmungsmuster mit Vorstellung vom Wesen der höheren Wirklichkeit einhergehen, besteht eine gewisse Überschneidung mit der ideologischen Dimension. Zugleich sind auch Überschneidungen zu den Dimensionen der privaten und öffentlichen Praxis vorhanden, da in 
ihnen bestimmte Gestalten der höheren Wirklichkeit transportiert werden. Gleichwohl kann die Eigenständigkeit der Dimension der religiösen Erfahrung festgehalten werden. Ihre Spezifik basiert auf dem Ereignischarakter religiöser Erfahrungen, den Wahrnehmungsmustern, die sie ermöglichen, und den Gefühlen, die damit verbunden sind.

In der CRS ist diese Kerndimension je nach Version durch bis zu drei Basisindikatoren operationalisiert (vgl. Huber und Huber 2012, S. 718):

1. Wie oft erleben Sie Situationen, in denen Sie das Gefühl haben, dass Gott oder etwas Göttliches in Ihr Leben eingreift?

2. Wie oft erleben Sie Situationen, in denen Sie das Gefühl haben, dass Gott oder etwas Göttliches Ihnen etwas sagen oder zeigen will?

3. Wie oft erleben Sie Situationen, in denen Sie das Gefühl haben, dass Gott oder etwas Göttliches anwesend ist?

Bei allen drei Indikatoren wird nach der Häufigkeit von Ereignissen gefragt, in denen ein Kontakt mit der höheren Wirklichkeit wahrgenommen wird. Dadurch wird die Formspezifik erreicht. Um die Abgrenzung zur ideologischen Dimension zu verstärken, wird darüber hinaus jeweils der Nebensatz ,, in denen Sie das Gefühl haben “ eingefügt. Diese soll verdeutlichen, dass es um eine Wahrnehmung geht und nicht um den ontologischen Status dieser Wahrnehmung oder um die Einstellung, ob es eine höhere Macht gibt, die mit Personen interagiert. Dass diese Unterscheidung auch empirisch relevant ist, zeigen z. B. die Daten des Religionsmonitors $2007 \mathrm{zu}$ Ostdeutschland. Während in dieser stark säkular geprägten Region 50\% der Respondenten angeben, dass sie gar nicht an Gott oder Göttliches glauben, geben zugleich $71 \%$ der Respondenten an, dass sie zumindest selten religiöse Erfahrungen machen (vgl. Huber 2011, S. 182). Die Differenz der Antworten erschiene nur dann inkonsistent, wenn Erfahrungen als Einstellungen verstanden würden. Wird demgegenüber der Wahrnehmungscharakter religiöser Erfahrungen betont, dann wird durch die Berücksichtigung der Dimension der religiösen Erfahrung ein substanzieller Erkenntnisgewinn realisiert.

Die interreligiöse Verständlich- und Anwendbarkeit der Indikatoren soll analog zur religiösen Ideologie durch die Präsentation der alternativen Konzepte Gott und Göttliches erreicht werden. Gleichermaßen wird in hinduistischen oder buddhistischen Kontexten empfohlen, als dritte Alternative Gottheiten zu ergänzen (vgl. Huber und Huber 2012, S. 719). Darüber hinaus wird in islamischen Kontexten empfohlen, die absolute Souveränität Gottes zu betonen, indem ,,in Ihr Leben eingreift“ und „Ihnen etwas sagen oder zeigen will“ durch ,in Ihr Leben eingreifen lässt“ und „Ihnen etwas sagen oder zeigen lässt“ ersetzt wird. Demmrich (2020) konnte zeigen, dass diese Stärkung der Souveränität Gottes auch bei Befragungen von Angehörigen der Religionsgemeinschaft der Bahai, die im 19. Jahrhundert im Iran aus dem Islam entstanden ist, empfohlen werden kann.

Die ersten beiden Indikatoren zur religiösen Erfahrung transportieren ein interaktives Wahrnehmungsmuster ab; d.h. Gott oder Göttliches wird als eine aktive Instanz wahrgenommen, die auf eine Person zugeht oder es zulässt, dass auf eine Person zugegangen wird. Daneben sind in allen großen religiösen Traditionen auch mystische Erfahrungen belegt, die als ein partizipatives Wahrnehmungsmuster ver- 
standen werden können. Dieser Typ religiöser Erfahrungen wird in der CRS durch zwei zusätzliche Indikatoren berücksichtigt (vgl. Huber und Huber 2012, S. 718):

1b. Wie oft erleben Sie Situationen, in denen Sie das Gefühl haben, mit allem eins zu sein?

2b. Wie oft erleben Sie Situationen, in denen Sie das Gefühl haben, dass Sie von einer göttlichen Kraft berührt werden?

Da die CRS auf die allgemeine Stärke der Ausprägung der verschiedenen Kerndimensionen zielt, werden für die Dimension der religiösen Erfahrung nicht getrennte Indizes für die Stärke interaktiver und partizipativer Wahrnehmungsmuster berechnet. Vielmehr wird bei Indikatoren 1 und $1 b$ sowie 2 und $2 b$ nur der jeweils höhere Wert gezählt. Dadurch soll die allgemeine Stärke der Erfahrungsdimension abgebildet werden - und zwar möglichst unabhängig vom Inhalt der religiösen Erfahrung. Auch das führt zu einer Erhöhung der interreligiösen Reichweite der CRS.

\subsubsection{Private religiöse Praxis}

Diese Kerndimension wird soziologisch durch die soziale Erwartung konstituiert, dass Angehörige von Religionsgemeinschaften sich regelmäßig an die höhere Wirklichkeit, an die sie glauben, wenden. Wesentlich ist dabei die personale Hinwendung zu dieser Wirklichkeit in einem privaten Raum. Die dafür typischste und sicher am weitesten verbreitete Form ist das Gebet. Es findet sich in allen Religionen, wenn auch mit religionsspezifischen Modifikationen in Bezug auf den Grad der Ritualisierung (Pflichtgebete, freie Gebete) und die Adressaten (Gott, Gottheiten, Bodhisattvas, Ahnen). Insbesondere im Islam ist die Unterscheidung zwischen Pflichtgebeten (Salāt) und freien Gebeten (Du'à') deutlich ausgeprägt. Im Hinduismus ist das Gebet eine gängige und häufig geübte Praxis (vgl. von Brück 2009, S. 326). Auch im Buddhismus ist das Gebet verbreitet, Adressaten sind insbesondere Gottheiten und Bodhisattvas.

In der CRS ist diese Kerndimension je nach Version durch bis zu drei Indikatoren operationalisiert, die sich auf die Häufigkeit und Wichtigkeit des Gebets beziehen (vgl. Huber und Huber 2012, S. 718):

1. Wie häufig beten Sie?

2. Wie wichtig ist für Sie das persönliche Gebet?

3. Wie oft richten Sie mitten in Ihrem Alltag ein kurzes Gebet an Gott?

Bei den drei Indikatoren wird das Gebet in möglichst allgemeiner Form angesprochen, um Verengungen auf Gebetskonzepte bestimmter Religionen zu vermeiden. Eine Ausnahme bilden Anwendungen der CRS in islamischen Kontexten. Hier wird empfohlen, zunächst nach der Häufigkeit des Pflichtgebets (Salāt) zu fragen und danach erst nach der Häufigkeit des freien Gebets (Du'ā'). Dadurch wird muslimischen Respondenten der Gegenstand der Frage klarer, was die Antwort erleichtert. In die Berechnung des Skalenwerts geht dann nur die Antwort auf das freie Gebet ein. Dies hat den Zweck, die Vergleichbarkeit mit Antworten aus anderen Religionen $\mathrm{zu}$ erhöhen. In hinduistischen, buddhistischen und schintoistischen Kontexten bietet es sich schließlich an, im dritten Indikator Gott durch Gottheiten zu ergänzen. 
Darüber hinaus wäre es auch möglich, Ahnen oder Geistwesen zu ergänzen, falls dies in einer bestimmten religiösen Kultur angebracht erscheint.

Das Gebet impliziert eine dialogische Struktur und ähnelt damit dem in der religiösen Erfahrung eingeführten interaktiven Wahrnehmungsmuster. In analoger Weise kann Meditation als eine private Praxis verstanden werden, die mit einem partizipativen Wahrnehmungsmuster korrespondiert. Um diese Form der privaten religiösen Praxis abbilden zu können, sind in der CRS auch bis zu drei Indikatoren zur Meditation integriert:

1b. Wie häufig meditieren Sie?

2b. Wie wichtig ist für Sie Meditation?

3b. Wie oft suchen Sie mitten in Ihrem Alltag Kontakt zu einer göttlichen Kraft?

Die Berechnung des Skalenwerts erfolgt bei der privaten religiösen Praxis in gleicher Weise wie bei der religiösen Erfahrung. Von den Indikatoren 1 und 1b, 2 und $2 \mathrm{~b}$ sowie 3 und $3 \mathrm{~b}$ wird nur der jeweils höhere Wert gezählt.

\subsection{5 Öffentliche religiöse Praxis}

Diese Kerndimension der öffentlichen Praxis wird soziologisch durch die soziale Erwartung konstituiert, dass Angehörige von Religionsgemeinschaften sich regelmäßig zu Ritualen versammeln, in denen die höhere Wirklichkeit gemeinschaftlich verehrt wird. Neben der Gemeinschaftlichkeit ist der öffentliche Raum, in dem diese Praxis stattfindet, wesentlich. Gemeinschaftlich vollzogene Rituale gibt es in allen großen Religionen. Daher stehen die Allgemeinheit und interreligiöse Relevanz dieser Dimension kaum infrage.

In der CRS ist diese Kerndimension je nach Version durch bis zu drei Indikatoren operationalisiert (vgl. Huber und Huber 2012, S. 718):

1. Wie häufig nehmen Sie an Gottesdiensten teil?

2. Wie wichtig ist Ihnen die Teilnahme an Gottesdiensten?

3. Wie wichtig ist Ihnen die Verbindung zu einer religiösen Gemeinschaft?

Bei Anwendungen in nicht-christlichen Kontexten wird der Begriff Gottesdienst der jeweiligen Religionskultur angepasst (z. B. Freitagsgebet im Islam, Synagogengottesdienst im Judentum, Tempelritual im Hinduismus).

\subsubsection{Konsequenzen im Alltag}

Die Kerndimension der Konsequenzen im Alltag wird soziologisch durch die soziale Erwartung konstituiert, dass Angehörige von Religionsgemeinschaften auch im Alltag Regeln befolgen, die von ihrer Religion vorgegeben werden. Wie bereits erwähnt, wurde die Dimension der Konsequenzen im Alltag nicht in die CRS integriert. Im Religiositäts-Struktur-Test (R-S-T) finden sich jedoch zwei Indikatoren, mit denen die allgemeine Intensität dieser Dimension erfasst werden kann (vgl. Huber 2008b, S. 133): 
1. Wie stark leben Sie in Ihrem Alltag nach religiösen Geboten?

2. Wie wichtig ist es für Sie, im Alltag religiöse Regeln zu beachten?

Da bei diesen Indikatoren keine Bezüge zu bestimmten Geboten hergestellt werden, sondern nur zu religiösen Geboten und Regeln im Allgemeinen, sollte auch bei diesen Indikatoren interreligiösen Anwendungen nichts im Wege stehen.

\section{Anwendungen in unterschiedlichen Religionskulturen}

Wie bereits in der Einleitung erwähnt, nehmen Anwendungen der CRS in nichtchristlichen Kontexten zu. Dies eröffnet die Chance, vertiefte Analysen zur Reliabilität und Validität der Skala in verschiedenen Religionskulturen durchzuführen sowie Weiterentwicklungsmöglichkeiten zu prüfen.

Für die religionsübergreifende Reliabilität der Skala sprechen bereits die Befunde der ersten Welle des internationalen Religionsmonitors, die in 21 Ländern sowie in Deutschland mit einer Zusatzbefragung von 2000 Muslimen durchgeführt wurde. Dazu sind in der Tab. 1 die Reliabilitäten (Cronbachs Alpha) der CRS-10 dokumentiert.

Die CRS-10 besteht aus zehn Indikatoren. Dabei werden die ersten beiden Indikatoren der ersten fünf Kerndimensionen verwendet. Als Daumenregel gilt, dass Alphakoeffizienten ab einem Wert von 0,65 gerade noch akzeptabel sind. Ab 0,70 werden sie als befriedigend bewertet, ab 0,80 als gut und ab 0,90 als sehr gut. Bei der Interpretation der Koeffizienten ist weiter zu berücksichtigen, dass aus mathematischen Gründen gute Koeffizienten erst ab einer gewissen Varianz erreicht werden können. Inhaltlich ist dies erst dann der Fall, wenn sowohl hochreligiöse Respondenten als auch nichtreligiöse Respondenten einen substanziellen Anteil in der Stichprobe haben. Sind jedoch beispielsweise die meisten Respondenten hochreligiös, dann kollabiert die Statistik. Dies ist in Nigeria der Fall, wo $89 \%$ der Muslime

Tab. 1 Cronbachs Alpha der CRS-10 in verschiedenen Ländern bei Angehörigen von fünf Religionskulturen (Daten des Religionsmonitors 2007)

\begin{tabular}{llllll}
\hline & Juden & Christen & Muslime & Hindus & Buddhisten \\
\hline Israel & 0,93 & - & - & - & - \\
Christllich-säkulare Länder & - & $0,88-0,93$ & 0,87 & - & - \\
Brasilien & - & 0,74 & - & - & - \\
Guatemala & - & 0,74 & - & - & - \\
Türkei & - & - & 0,72 & - & - \\
Marokko & - & - & 0,68 & - & - \\
Indonesien & - & - & 0,68 & - & - \\
Nigeria & - & 0,57 & 0,57 & - & - \\
Indien & - & - & - & 0,65 & - \\
Thailand & - & - & - & - & 0,72 \\
Südkorea & - & 0,89 & - & - & 0,88 \\
\hline
\end{tabular}

Zu christlich-säkularen Ländern zählen Deutschland, Österreich, Schweiz, Italien, Frankreich, Spanien, UK, USA, Australien, Russland und Polen 
und $91 \%$ der Christen aufgrund ihres CRS-Werts als hochreligiös klassifiziert werden können. Daher ist es nicht überraschend, dass der Alphakoeffizient in Nigeria mit 0,57 nicht akzeptabel ist. Dies ist sowohl für Muslime als auch für Christen der Fall.

Wie erwähnt, kann ein guter Alphakoeffizient erst dann erreicht werden, wenn genügend säkulare oder nichtreligiöse Personen in einer Stichprobe vorhanden sind. Dies ist beim Religionsmonitor neben den christlich-säkularen Ländern nur in Israel und in Südkorea der Fall. Unter dieser Voraussetzung erreicht die CRS bei Juden, Christen, Muslimen und Buddhisten gute bis sehr gute Reliabilitäten, die zwischen 0,87 und 0,93 schwanken. Dies spricht für die interreligiöse Anwendbarkeit der Skala. Besonders aussagekräftig sind die Befunde in Südkorea, weil in diesem Land etwa gleich viele Buddhisten und Christen befragt wurden. Wie Tab. 1 belegt, unterschieden sich die Reliabilitätskoeffizienten bei Angehörigen der beiden Religionsgemeinschaften in diesem Land kaum (Christen: 0,89; Buddhisten: 0,88). Dies ist ein starkes Indiz dafür, dass Angehörige beider Religionskulturen gleich gut mit den Semantiken der Indikatoren der CRS arbeiten können.

Befriedigende Alphakoeffizienten finden sich für Christen in Brasilien und Guatemala, Muslime in der Türkei und Buddhisten in Thailand $(0,72-0,74)$. Diesen Ländern ist gemeinsam, dass sie (mit Ausnahme von Guatemala) im Jahr 2007 einen ähnlich hohen Human Development Index (HDI) von 0,78 bis 0,80 erreichten (zum HDI vgl. Stanton 2007). Gerade noch akzeptabel sind die Alphakoeffizienten bei Hindus in Indien und bei Muslimen in Marokko und Indonesien $(0,65-0,68)$, wobei Indien und Marokko im Jahr 2007 mit einem HDI von 0,61 und 0,63 ein besonders niedriges Entwicklungsniveau erreicht hatten. Niedriger war nur Nigeria mit einem HDI von 0,47. Diese Befunde deuten darauf hin, dass befriedigende und gerade noch akzeptable Reliabilitäten der CRS mehr vom wirtschaftlichen Entwicklungs- und Modernisierungsniveau sowie dem Anteil von Säkularen abhängen als von der Zugehörigkeit zu einer bestimmten Religionskultur.

Zusammenfassend kann somit festgehalten werden, dass sich die CRS-10 mit der Ausnahme von Muslimen und Christen in Nigeria in allen Ländern und Religionskulturen des internationalen Religionsmonitors 2007 als reliabel erwiesen hat. Mit ihr kann die Zentralität der Religiosität in allen großen Religionskulturen abgebildet werden. Daher eignet sich die Skala als tertium comparationis für religionsvergleichende Studien (vgl. Huber und Krech 2009).

Offen bleiben die Fragen nach Wechselwirkungen, Überschneidungen und relativem Gewicht der fünf bzw. sechs Kerndimensionen in unterschiedlichen Religionskulturen. Dies kann in konfirmatorischen Faktorenanalysen und Strukturgleichungsmodellen untersucht werden. Sarah Demmrich (2020) hat eine derartige Analyse mit Daten von Angehörigen der Religionsgemeinschaft der Baha'i durchgeführt. Dabei konnte sie die Reliabilität und Validität der CRSi-20 für Anwendungen in dieser Religionskultur belegen. Michael Ackert et al. (2020, im Druck, in Vorbereitung) haben ausführliche Analysen zur CRS-5 und zur CRSi-7 bei Angehörigen christlich-orthodoxer Kirchen in Russland, Rumänien und Georgien durchgeführt. Dabei bestätigte sich die Reliabilität und Validität der CRS für die christlich-orthodoxe Religionskultur. Darüber hinaus zeigte sich als ein durchgängiges Merkmal, dass der Zusammenhang zwischen der privaten und öffentlichen religiösen Praxis we- 
sentlich höher ist als zwischen den anderen Dimensionen der Religiosität. Dieses Ergebnis sollte mit Daten anderer christlicher Konfessionen und anderer Religionsgemeinschaften verglichen werden. Leider stehen analoge Studien mit jüdischen, islamischen, hinduistischen und buddhistischen Respondenten noch aus.

Für ein tieferes Verständnis der Relevanz und Funktionsweise der oben definierten Kerndimensionen der Religiosität sind qualitative Untersuchungen unerlässlich. Dabei können die inhaltliche Füllung, das relative Gewicht und die Wechselwirkungen dieser Dimensionen in unterschiedlichen Religionskulturen untersucht werden. Ein erster Schritt in diese Richtung ist die Studie, die Demmrich und Huber (2019) an säkularen Personen in der Schweiz durchgeführt haben. Dabei zeigte sich, dass die sechs Kerndimensionen ausreichen, um fast alle spirituellen und religiösen Inhalte, die in einem qualitativen Interview geäußert werden, zu kodieren. Dies spricht für die Validität dieses Modells der Religiosität.

Funding Open access funding provided by University of Bern

Open Access Dieser Artikel wird unter der Creative Commons Namensnennung 4.0 International Lizenz veröffentlicht, welche die Nutzung, Vervielfältigung, Bearbeitung, Verbreitung und Wiedergabe in jeglichem Medium und Format erlaubt, sofern Sie den/die ursprünglichen Autor(en) und die Quelle ordnungsgemäß nennen, einen Link zur Creative Commons Lizenz beifügen und angeben, ob Änderungen vorgenommen wurden.

Die in diesem Artikel enthaltenen Bilder und sonstiges Drittmaterial unterliegen ebenfalls der genannten Creative Commons Lizenz, sofern sich aus der Abbildungslegende nichts anderes ergibt. Sofern das betreffende Material nicht unter der genannten Creative Commons Lizenz steht und die betreffende Handlung nicht nach gesetzlichen Vorschriften erlaubt ist, ist für die oben aufgeführten Weiterverwendungen des Materials die Einwilligung des jeweiligen Rechteinhabers einzuholen.

Weitere Details zur Lizenz entnehmen Sie bitte der Lizenzinformation auf http://creativecommons.org/ licenses/by/4.0/deed.de.

\section{Literatur}

Ackert, M., E. Maglakelidze, I. Badurashvili, und S. Huber. 2020. Validation of the short forms of the centrality of religiosity scale in Georgia. Religions https://doi.org/10.3390/rel11020057.

Ackert, M., S. Huber, A.P. Plopeanu, und I. Zabaev. in Vorbereitung. Validation of the short forms of the centrality of religiosity scale in Romania (Arbeitstitel). Religions 11.

Ackert, M., S. Huber, E. Prutskova, und I. Zabaev. im Druck. Validation of the short forms of the centrality of religiosity scale in Russia. Religions 11.

Demmrich, S. 2020. How to measure Baha'i religiosity: the CRsi-20 for Baha'is as a first reliable and valid measurement. Religions https://doi.org/10.3390/rel11010029.

Demmrich, S., und S. Huber. 2019. Multidimensionality of spirituality: a qualitative study among secular individuals. Religions https://doi.org/10.3390/rel10110613.

Glock, C.Y. 1962. On the study of religious commitment. Review of recent research bearing on religious and character formation. Religious Education 57(Research Supplement):98-110.

Huber, S. 2003. Zentralität und Inhalt. Ein neues multidimensionales Messmodell der Religiosität. Opladen: Leske + Budrich.

Huber, S. 2004. Zentralität und multidimensionale Struktur der Religiosität: Eine Synthese der theoretischen Ansätze von Allport und Glock zur Messung der Religiosität. In Religiosität: Messverfahren und Studien zu Gesundheit und Lebensbewältigung, Hrsg. C. Zwingmann, H. Moosbrugger, 79-105. Münster/New York/München/Berlin: Waxmann.

Huber, S. 2007. Are religious beliefs relevant in daily life? In Religion Inside and Outside Traditional Institutions, Hrsg. H. Streib, 211-230. Leiden/Boston: Brill. 
Huber, S. 2008a. Kerndimensionen, Zentralität und Inhalt. Ein interdisziplinäres Modell der Religiosität. Journal für Psychologie 16(3). https://doi.org/10.7892/boris.73105. https://www.journal-fuerpsychologie.de/index.php/jfp/article/view/202.

Huber, S. 2008b. Der Religiositäts-Struktur-Test (R-S-T). Systematik und operationale Konstrukte. In Individualisierung - Spiritualität - Religion: Transformationsprozesse auf dem religiösen Feld in interdisziplinärer Perspektive, Hrsg. W. Gräb, L. Charbonnier, 131-171. Münster: Lit.

Huber, S. 2009. Der Religionsmonitor 2008: Strukturierende Prinzipien, operationale Konstrukte, Auswertungsstrategien. In Woran glaubt die Welt? Analysen und Kommentare zum Religionsmonitor 2008, Hrsg. Bertelsmann Stiftung, 17-52. Gütersloh: Bertelsmann Stiftung.

Huber, S. 2011. Religiosität und Spiritualität im deutschsprachigen Raum. Ein Überblick. In Die Bedeutung von Religion für die Gesundheit. Konzepte - Befunde - Erklärungsansätze, Hrsg. F. Balck, H. Berth, und C. Klein, 163-187. Weinheim: Juventa.

Huber, S., und O.W. Huber. 2012. The centrality of religiosity scale (CRS). Religions 3(3):710-724. https:// doi.org/10.3390/rel3030710.

Huber, S., und V. Krech. 2009. Das religiöse Feld zwischen Globalisierung und Regionalisierung: Vergleichende Perspektiven. In Woran glaubt die Welt? Analysen und Kommentare zum Religionsmonitor 2008, 53-96. Gütersloh: Bertelsmann Stiftung.

Pollack, D. 2000. Was ist Religion? In Die religiöse Dimension im Geschichtsunterricht an Europas Schulen. Ein interdisziplinäres Forschungsprojekt, Hrsg. W. Schreiber, 55-81. Neuried: Ars una.

Riesebrodt, M. 2007. Cultus und Heilsversprechen. Eine Theorie der Religionen. München: Beck.

Schlieter, J. 2001. Buddhismus zur Einführung. Hamburg: Junius.

Stanton, E.A. 2007. The human development index: a history. Working paper series, Bd. 127. Amhers: Political Economy Research Institute, University of Massachusetts. http://www.peri.umass.edu/ fileadmin/pdf/working_papers/working_papers_101-150/WP127.pdf.

Stark, R., und C.Y. Glock. 1970. American piety: the nature of religious commitment. Berkeley/Los Angeles: University of California Press.

Von Brück, M. 2007. Einführung in den Buddhismus. Frankfurt a. Main/Leipzig: Verlag der Weltreligionen.

Von Brück, M. 2009. Zur gegenwärtigen Entwicklung der Religion(en) in Indien. In Woran glaubt die Welt? Analysen und Kommentare zum Religionsmonitor 2008, 313-328. Gütersloh: Bertelsmann Stiftung. 